\title{
Phase space tomography reconstruction of the Wigner distribution for optical beams separable in Cartesian coordinates
}

\author{
Alejandro Cámara, ${ }^{1, *}$ Tatiana Alieva, ${ }^{1}$ José A. Rodrigo, ${ }^{2}$ and María L. Calvo ${ }^{1}$ \\ ${ }^{1}$ Universidad Complutense de Madrid, Facultad de Ciencias Físicas, Ciudad Universitaria s/n, Madrid 28040, Spain \\ ${ }^{2}$ Instituto de Óptica (CSIC), Imaging and Vision Department, Serrano 121, Madrid 28006, Spain \\ *Corresponding author: a.camara@fis.ucm.es
}

Received February 10, 2009; revised April 2, 2009; accepted April 8, 2009; posted April 9, 2009 (Doc. ID 107376); published May 7, 2009

\begin{abstract}
We propose a simple approach for the phase space tomography reconstruction of the Wigner distribution of paraxial optical beams separable in Cartesian coordinates. It is based on the measurements of the antisymmetric fractional Fourier transform power spectra, which can be taken using a flexible optical setup consisting of four cylindrical lenses. The numerical simulations and the experimental results clearly demonstrate the feasibility of the proposed scheme. (C) 2009 Optical Society of America

OCIS codes: $070.2575,100.5070,120.4820$.
\end{abstract}

\section{INTRODUCTION}

The determination of the phase or mutual intensity of coherent or partially coherent monochromatic optical fields, respectively, is required for different applications of optical signals, such as phase imaging, beam characterization, and communication. Different approaches, including interferometric and iterative techniques, have been proposed for this task [1-15].

More than ten years ago, a noninterferometric, noniterative method [5,6] for the reconstruction of the Wigner distribution (WD) of optical fields, called phase space tomography, was established. It can be applied for the characterization of coherent and partially coherent fields. The phase space tomography method is based on the rotation of the Wigner distribution during the fractional Fourier transform (FRFT) [16] and also on the fact that the WD projections coincide with the intensity distribution (FRFT power spectra) measured at the output plane of the optical system performing the FRFT. The main obstacle to its wide implementation is the lack of flexible optical setups that allow one to make the required experimental measurements. Indeed, for tomographic reconstruction of the Wigner distribution of a one-dimensional signal, the WD projections for different angles $\alpha$ have to be measured. Two simple setups have been proposed for optical FRFT implementation [17], consisting of either one or two convergent lenses. Nevertheless, for the scanning of angle $\alpha$, the use of lenses with different focal lengths and/or changes of the distances between the lens and the input/ output planes is required. Moreover, the measured intensity distributions need some rescaling, which depends on $\alpha$, in order to be used in the reconstruction algorithm. A similar setup was applied to obtain the Radon-Wigner maps of a one-dimensional field [18]. In the case of twodimensional signals the systems become even more complicated $[5,6]$. For the acquisition of a large number of WD projections, an optical setup performing the FRFT with easily changeable angular parameters is required.

Recently, lens-based optical systems able to perform image rotation, FRFT, and gyrator transform for tunable angles have been designed [19]. In particular, the experimental realization of the gyrator transform has been reported in [20]. The same setup rotated at angle $\pi / 4$ performs the two-dimensional FRFT for angles $(\alpha,-\alpha)$, which will be denoted further on as antisymmetric FRFT for angle $\alpha$. This optical scheme contains three generalized lenses $\mathrm{L}_{1}, \mathrm{~L}_{2}$, and $\mathrm{L}_{3}=\mathrm{L}_{1}$ with fixed identical distances between them, denoted by $z$. Every generalized lens $\mathrm{L}_{j}$, where $j=1,2$, is constructed from two convergent cylindrical lenses with the same focal distance $\mathrm{f}_{j}=z / j$. The transformation angle $\alpha$ is changed by rotation of the cylindrical lenses at angle $\phi_{j} \pm \pi / 4$ with respect to the transverse axes OX, where $2 \sin \left(2 \phi_{1}\right)=\cot (\alpha / 2)$ and $2 \phi_{2}=\alpha$. Since only the measurements of the FRFT power spectra are required for the WD reconstruction, a CCD camera is placed at the output plane instead of the third generalized lens, $\mathrm{L}_{3}$, as displayed in Fig. 1. The system allows measurement of the antisymmetric FRFT power spectra for angles in the interval $\alpha \in[\pi / 2,3 \pi / 2]$.

In this paper we demonstrate that this flexible setup can be used for the reconstruction of the WD of twodimensional optical beams separable in Cartesian coordinates as well as the reconstruction of one-dimensional fields, used, for example, in planar optics.

The paper is organized as follows. Section 2 is devoted to the consideration of the basic properties of the FRFT power spectra of separable fields. In Section 3 we propose an algorithm for the reconstruction of the WD of the separable beams from the measurements of the antisymmetric FRFT spectra. The feasibility of this method is demonstrated by numerical simulations and experimental results in Section 4. 


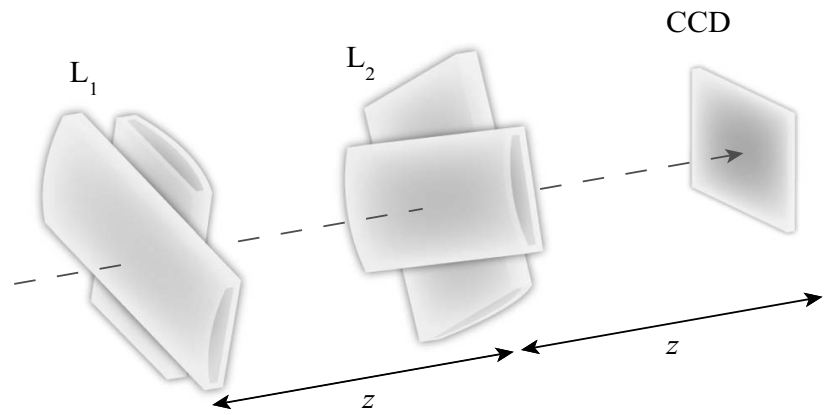

Fig. 1. Scheme of the optical system for the antisymmetric FRFT power spectra measurements. The parameter $\alpha$ is varied by rotating the cylindrical lens while the free-space intervals $z$ are fixed. The FRFT power spectra are registered by a CCD camera (WXGA, pixel size $4.6 \mu \mathrm{m}$ ).

\section{FRACTIONAL FOURIER TRANSFORM POWER SPECTRA OF SEPARABLE BEAMS}

The complex field amplitude at the output plane of the optical system performing the two-dimensional FRFT at angles $\alpha_{x}$ and $\alpha_{y}$ is defined as

$$
F^{\alpha_{x}, \alpha_{y}}\left(\mathbf{r}_{o}\right)=\iint f\left(\mathbf{r}_{i}\right) K^{\alpha_{x}, \alpha_{y}}\left(\mathbf{r}_{i}, \mathbf{r}_{o}\right) \mathrm{d} \mathbf{r}_{i}
$$

where $f\left(\mathbf{r}_{i}\right)$ is the complex field amplitude at the input plane, $\mathbf{r}=(x, y)^{t}$ are the spatial coordinates at the input and output planes indicated by subindices $i$ and $o$, respectively, and $t$ stands for transposition operator. The kernel $K^{\alpha_{x}, \alpha_{y}}$ is separable in Cartesian coordinates $K^{\alpha_{x}, \alpha_{y}}$ $=K^{\alpha_{x}} K^{\alpha_{y}}$, where $K^{\alpha_{q}}$ for $\alpha_{q} \neq \pi n$ is given by [16]

$$
K^{\alpha} q\left(q_{i}, q_{o}\right)=\left(i s \sin \alpha_{q}\right)^{-1 / 2} \exp \left[i \pi \frac{\left(q_{i}^{2}+q_{o}^{2}\right) \cos \alpha_{q}-2 q_{i} q_{o}}{s \sin \alpha_{q}}\right]
$$

and $q$ is a placeholder for $x$ or $y$. The parameter $s$ has the dimension of length squared, and its value depends on the optical system used. For $\alpha_{q}=\pi n$, the kernel is $\delta\left(q_{i}+q_{o}\right)$ or $\delta\left(q_{i}-q_{o}\right)$ for odd or even $n$, respectively. It is easy to see that the kernel $K^{\alpha} q\left(q_{i}, q_{o}\right)$ reduces to the common direct (inverse) FT for $\alpha_{q}= \pm \pi / 2$ apart from a constant phase $\sqrt{\mp i}$. If $\alpha_{x}=-\alpha_{y}$ is set in Eq. (1), the antisymmetric FRFT is obtained. Also note that $K^{-\alpha_{q}}=\left(K^{\alpha_{q}}\right)^{*}$.

The FRFT power spectrum of $f\left(\mathbf{r}_{i}\right)$ at angles $\alpha_{x}, \alpha_{y}$, which is associated with the intensity distribution of the optical field at the output plane of the fractional Fourier transformer, is defined as

$$
S_{f}^{\alpha_{x}, \alpha_{y}}\left(\mathbf{r}_{o}\right)=\left|\iint f\left(\mathbf{r}_{i}\right) K^{\alpha_{x}, \alpha_{y}}\left(\mathbf{r}_{i}, \mathbf{r}_{o}\right) \mathrm{d} \mathbf{r}_{i}\right|^{2} .
$$

In the case of partially coherent light the ensemble averaging has to be introduced. That leads to

$$
\begin{aligned}
S_{f}^{\alpha_{x}, \alpha_{y}}\left(\mathbf{r}_{o}\right)= & \iiint \int \Gamma\left(\mathbf{r}_{i}, \mathbf{r}_{i}^{\prime}\right) K^{\alpha_{x}, \alpha_{y}}\left(\mathbf{r}_{i}, \mathbf{r}_{o}\right) \\
& \times\left[K^{\alpha_{x}, \alpha_{y}}\left(\mathbf{r}_{i}^{\prime}, \mathbf{r}_{o}\right)\right] * \mathrm{~d} \mathbf{r}_{i} \mathrm{~d} \mathbf{r}_{i}^{\prime},
\end{aligned}
$$

where $\Gamma\left(\mathbf{r}_{i}, \mathbf{r}_{i}^{\prime}\right)=\left\langle f\left(\mathbf{r}_{i}\right) f^{*}\left(\mathbf{r}_{i}^{\prime}\right)\right\rangle$ is the mutual intensity. Further, this definition is used because it reduces to Eq. (3) for coherent fields. In both cases, the quasimonochromatic approximation is applied.

If the input mutual intensity is separable with respect to the $x$ and $y$ variables, $\Gamma\left(\mathbf{r}_{i}, \mathbf{r}_{i}^{\prime}\right)=\Gamma_{x}\left(x_{i}, x_{i}^{\prime}\right) \Gamma_{y}\left(y_{i}, y_{i}^{\prime}\right)$, where $\Gamma_{q}\left(q, q^{\prime}\right)=\left\langle f_{q}(q) f_{q}^{*}\left(q^{\prime}\right)\right\rangle$, then, as observed from Eq. (4), the FRFT spectra are also separable:

$$
\begin{aligned}
S_{f}^{\alpha_{x}, \alpha_{y}}\left(\mathbf{r}_{o}\right)= & \iint \Gamma_{x}\left(x_{i}, x_{i}^{\prime}\right) K^{\alpha_{x}}\left(x_{i}, x_{o}\right) K^{-\alpha_{x}}\left(x_{i}^{\prime}, x_{o}\right) \mathrm{d} x_{i} \mathrm{~d} x_{i}^{\prime} \\
& \times \int \Gamma_{y}\left(y_{i}, y_{i}^{\prime}\right) K^{\alpha_{y}}\left(y_{i}, y_{o}\right) K^{-\alpha_{y}}\left(y_{i}^{\prime}, y_{o}\right) \mathrm{d} y_{i} \mathrm{~d} y_{i}^{\prime} \\
= & S_{f_{x}}^{\alpha_{x}}\left(x_{o}\right) S_{f_{y}}^{\alpha_{y}}\left(y_{o}\right) .
\end{aligned}
$$

Using the Parseval theorem for the one-dimensional FRFT [16],

$$
\int S_{f_{y}}^{\alpha}\left(y_{o}\right) \mathrm{d} y_{o}=\int S_{f_{y}}^{\beta}\left(y_{o}\right) \mathrm{d} y_{o}=A_{y}
$$

which indicates the energy conservation law, we derive that

$$
\int S_{f}^{\alpha_{x}, \alpha_{y}}\left(\mathbf{r}_{o}\right) \mathrm{d} y_{o}=A_{y} S_{f_{x}}^{\alpha_{x}}\left(x_{o}\right)
$$

for any angle $\alpha_{y}$, where $A_{y}$ is a constant. As we will see in the next section, this is a fundamental equation that permits the reconstruction of the mutual intensity (separable in Cartesian coordinates) by applying the flexible setup mentioned in the Introduction for the antisymmetric FRFT power spectra measurements.

Note that the Parseval relation can also be used to test the field separability. Indeed, only for fields that are separable in Cartesian coordinates, it holds that

$$
\int S_{f}^{\alpha_{x}, \alpha_{y}}(\mathbf{r}) \mathrm{d} y \int S_{f}^{\alpha_{x}, \alpha_{y}}(\mathbf{r}) \mathrm{d} x=S_{f}^{\alpha_{x}, \alpha_{y}}(\mathbf{r}) \int S_{f}^{\alpha_{x}, \alpha_{y}}(\mathbf{r}) \mathrm{d} x \mathrm{~d} y .
$$

Thus, based on the measurements of the antisymmetric FRFT power spectra needed for the reconstruction of the WD, the correctness of the hypothesis about the separability of the field in Cartesian coordinates can be proved.

\section{RECONSTRUCTION OF THE WIGNER DISTRIBUTION}

The WD is the Fourier transform of the mutual intensity of the optical field [21], and it is defined as

$$
W_{f}(\mathbf{r}, \mathbf{p})=C \iint \Gamma\left(\mathbf{r}+\mathbf{r}^{\prime} / 2, \mathbf{r}-\mathbf{r}^{\prime} / 2\right) \exp \left(-i 2 \pi \mathbf{p} \cdot \mathbf{r}^{\prime}\right) \mathrm{d} \mathbf{r}^{\prime},
$$

where $C$ is a normalization constant, $\mathbf{r}=(x, y)^{t}$ denote the transverse spatial coordinates, and $\mathbf{p}=\left(p_{x}, p_{y}\right)^{t}$ are spatial frequencies. These vectors have dimensions of length and inverse of length for $\mathbf{r}$ and $\mathbf{p}$, respectively. For coherent fields, the mutual intensity, $\Gamma$, is reduced to the product of the complex field amplitudes: $f\left(\mathbf{r}+\mathbf{r}^{\prime} / 2\right) f^{*}\left(\mathbf{r}-\mathbf{r}^{\prime} / 2\right)$. 
The WD is a real-valued function, and its integration over both variables, $\mathbf{r}$ and $\mathbf{p}$, is proportional to the radiation power. Moreover, the integration of the WD over $\mathbf{p}$ leads to the WD projection,

$$
S_{f}^{0,0}(\mathbf{r})=C \Gamma(\mathbf{r}, \mathbf{r})=\iint W_{f}(\mathbf{r}, \mathbf{p}) \mathrm{d} \mathbf{p},
$$

which is associated with the intensity distribution and corresponds to the FRFT power spectra at angles $\alpha_{x}=\alpha_{y}$ $=0$.

It is easy to see that for the mutual intensity separable in Cartesian coordinates, the WD is also separable: $W_{f}(\mathbf{r}, \mathbf{p})=W_{f_{x}}\left(x, p_{x}\right) W_{f_{y}}\left(y, p_{y}\right)$. Therefore, similarly to Eq. (6), we have

$$
\iint W_{f_{x}}\left(x, p_{x}\right) W_{f_{y}}\left(y, p_{y}\right) \mathrm{d} y \mathrm{~d} p_{y}=A_{y} W_{f_{x}}\left(x, p_{x}\right) .
$$

The problem of the WD reconstruction is then reduced to the one-dimensional case.

The rotation of the WD under the FRFT $[16,17]$ and the fact that the WD projections coincide with the intensity distribution (FRFT power spectra) measured at the output plane of the FRFT system, i.e.,

$$
\begin{aligned}
S_{f_{q}}^{\alpha_{q}}(q)= & \int W_{f_{q}}\left(q \cos \alpha_{q}-s p_{q} \sin \alpha_{q}, s^{-1} q \sin \alpha_{q}\right. \\
& \left.+p_{q} \cos \alpha_{q}\right) \mathrm{d} p_{q},
\end{aligned}
$$

lead to the conclusion that $S_{f}^{\alpha_{q}}(q)$ corresponds to the Radon transform of the WD [22]. Then, the WD can be reconstructed from the FRFT spectra, known for angles $\alpha$ from a $\pi$ interval, using the inverse Radon transform [23].

The proposed method of the reconstruction of the separable WD using the flexible setup for the antisymmetric FRFT spectra measurements consists of the following steps:

1. The measurement of the antisymmetric FRFT spectra, $S_{f}^{\alpha,-\alpha}(\mathbf{r})$, for angles $\alpha \in[\pi / 2,3 \pi / 2]$.

2. The integration of the acquired spectra over the $x$ or $y$ coordinate to obtain $A_{y} S_{f_{x}}^{\alpha}(x)$ or $A_{x} S_{f_{y}}^{-\alpha}(y)$ for angles $\alpha \in[\pi / 2,3 \pi / 2]$.

3 . The validation of the hypothesis about the field separability, which consists of the proof of Eq. (8) for $\alpha_{x}=-\alpha_{y}=\alpha$.

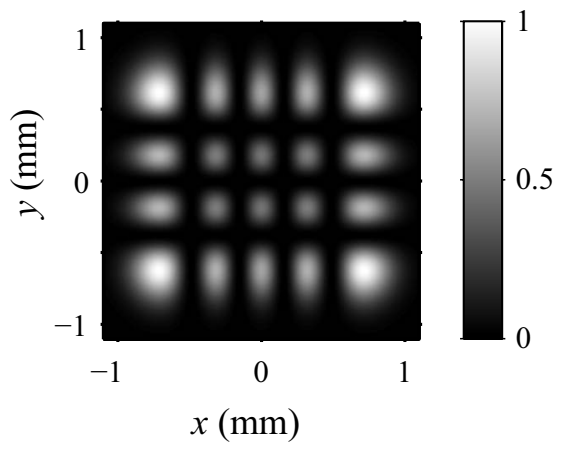

(a)
4. The application of the inverse Radon transform for the reconstruction of the two parts of the WD, $W_{f_{x}}\left(x, p_{x}\right)$ and $W_{f_{y}}\left(y, p_{y}\right)$, from $S_{f_{x}}^{\alpha}(x)$ and $S_{f_{x}}^{-\alpha}(y)$, correspondingly.

Note that, applying the inverse FT to the reconstructed $\mathrm{WD}$, it is possible to obtain the complex field amplitude, up to a constant phase factor, or the mutual intensity for the case of coherent or partially coherent light, respectively. The method can also be applied for the coherentstate estimation of one-dimensional signals.

\section{NUMERICAL SIMULATIONS AND EXPERIMENTAL RESULTS}

The feasibility of the method is demonstrated comparing numerically simulated examples with the experimental results. As a test field, the Hermite-Gaussian (HG) beam has been chosen,

$$
\mathcal{H}_{43}(x, y)=\mathcal{H}_{4}(x) \mathcal{H}_{3}(y),
$$

where $\mathcal{H}_{m}(q)$ are the well-known one-dimensional HG functions, defined by

$$
\begin{aligned}
\mathcal{H}_{n}(q)= & \frac{h}{\left(2^{n} n !\right)^{1 / 2}} H_{n}\left(\sqrt{2 \pi} \frac{q}{w}\right) \exp \left[-\pi\left(\frac{q}{w}\right)^{2}\right] \\
& \times \exp \left[-i \pi \frac{q^{2}}{\lambda R}\right],
\end{aligned}
$$

$w$ being the scale, $R$ the curvature of the mode, $\lambda$ the wavelength, $h$ a normalization constant, and $H_{n}(\cdot)$ the Hermite polynomial of degree $n$. This field is separable in $x$ and $y$ coordinates. Moreover, the intensity distributions of these beams preserve their form, apart from a separable scaling, during the propagation through the FRFT optical system. This property can be exploited to qualitatively check the performance of the experimental system. The intensity distribution and the phase of the $\mathcal{H}_{43}(x, y)$ field are displayed in Fig 2.

To ensure the validity of the phase tomography method for a restricted number of projections $N$, we performed numerical simulations. Calculating numerically the $N$ $=45$ antisymmetric FRFT spectra for the interval $\alpha$ $\in[\pi / 2,3 \pi / 2]$ (further used in the experiment), integrating the acquired spectra over the $y$ coordinate, and then applying the inverse Radon transform, we reconstructed



(b)

Fig. 2. (a) Intensity distribution and (b) phase of the $\mathcal{H}_{43}(x, y)$ field. In our case a curvature $R=2 \mathrm{~m}$ and a scale $w=0.75 \mathrm{~mm}$ have been used. 


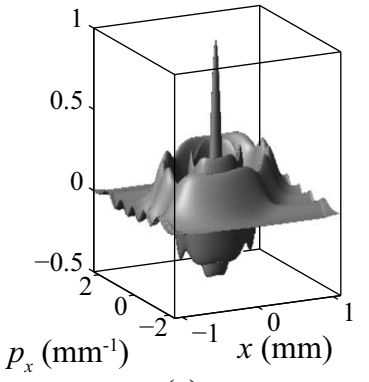

(a)

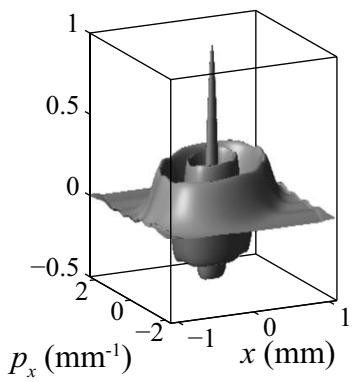

(b)

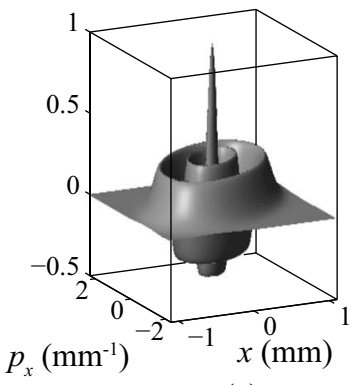

(c)

Fig. 3. WD of the $\mathcal{H}_{4}(x)$ mode reconstructed from the simulated (a) $N=45$ and (b) $N=180$ projections. (c) Theoretically calculated WD .

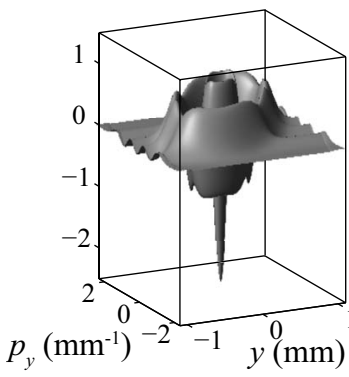

(a)

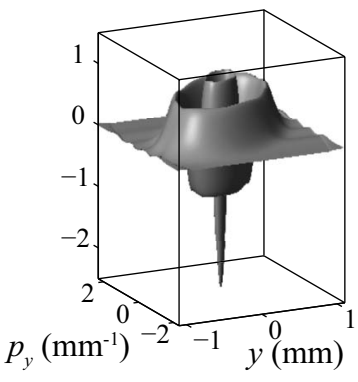

(b)

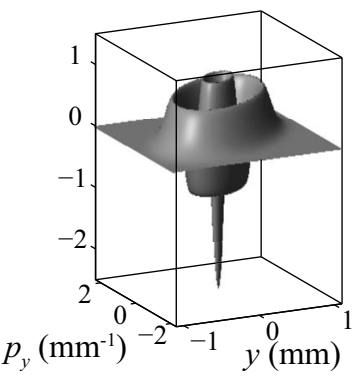

(c)

Fig. 4. WD of the $\mathcal{H}_{3}(y)$ mode reconstructed from the simulated (a) $N=45$ and (b) $N=180$ projections. (c) Theoretically calculated WD.

the WD of $\mathcal{H}_{4}(x)$, which is displayed in Fig. 3(a). The simulated WD and the one calculated directly from the definition, Eq. (9), which is shown in Fig. 3(c), are similar. In particular, the negative part of the simulated WD is clearly observed. If the number of the projections (FRFT spectra) increases to $N=180$ (one projection per degree), Fig. 3(b), then the difference between the simulation and the theoretical solutions becomes even smaller.

This agreement is also found for the $\mathcal{H}_{3}(y)$ part of the separable field as seen in Fig. 4, where the WDs recon- structed from $N=45$ [Fig. 4(a)] and $N=180$ [Fig. 4(b)] projections and calculated from its definition [Fig. 4(c)], are shown.

For experimental verification of the proposed scheme, the flexible setup mentioned in the Introduction has been used for the antisymmetric FRFT power spectra measurements. In the experiment we use a Nd:YAG laser as the coherent light source with wavelength $\lambda=532 \mathrm{~nm}$. The fixed free-space intervals (see Fig. 1) are set at $z=0.5 \mathrm{~m}$. The parameter $s$, defined for the FRFT system in Eq. (2),

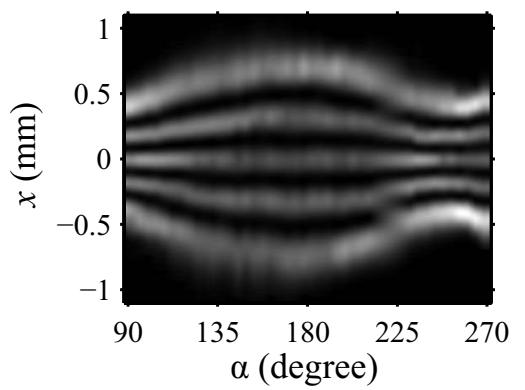

(a)

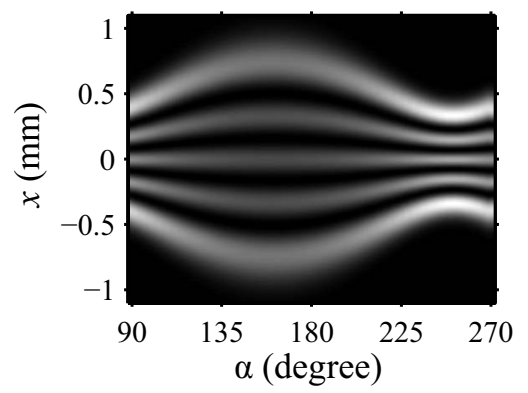

(c)

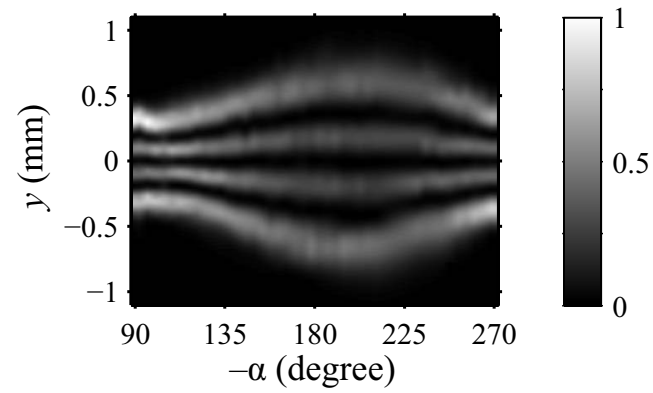

(b)

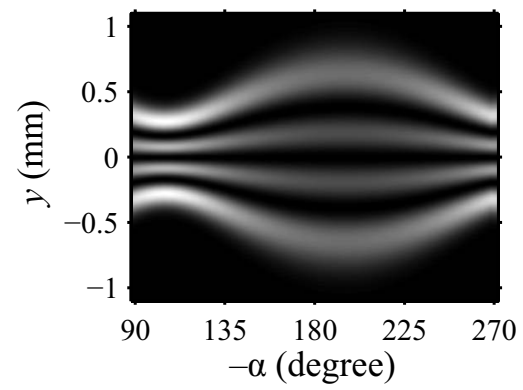

(d)

Fig. 5. Radon-Wigner maps for $N=45$ projections obtained experimentally for (a) $\mathcal{H}_{4}(x)$ and (b) $\mathcal{H}_{3}(y)$ and by simulation for the same modes, (c) and (d), respectively. 


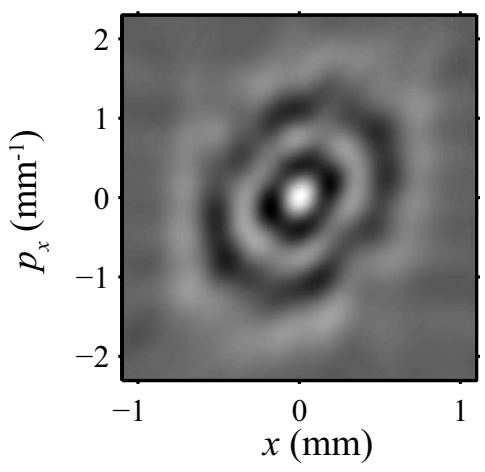

(a)

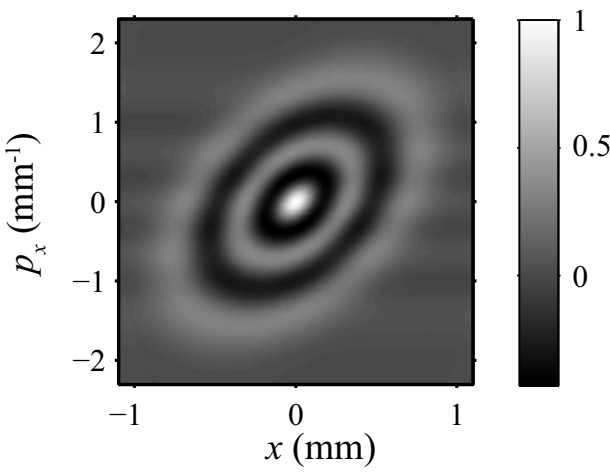

(b)

Fig. 6. WD of the $\mathcal{H}_{4}(x)$ mode reconstructed from $N=45$ (a) experimental and (b) simulated FRFT power spectra.

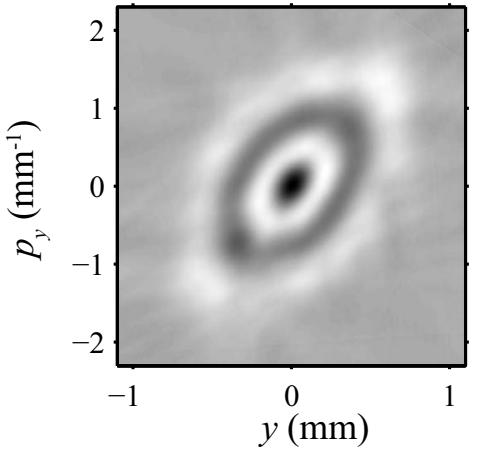

(a)

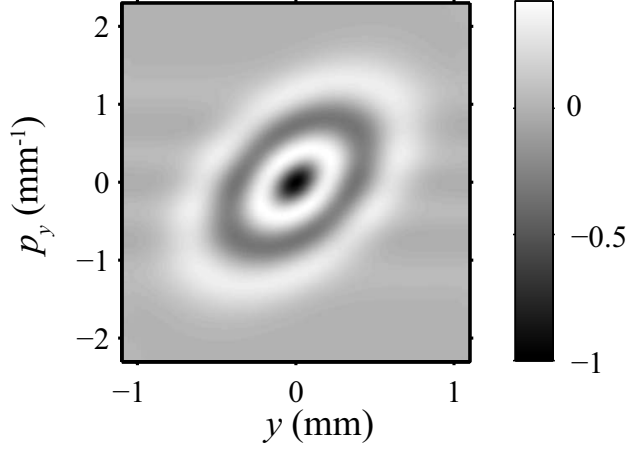

(b)

Fig. 7. WD of the $\mathcal{H}_{3}(y)$ mode reconstructed from $N=45$ (a) experimental and (b) simulated FRFT power spectra.

is $s=\lambda z=0.266 \mathrm{~mm}^{2}$. Meanwhile, the scale of the input signal, $\mathcal{H}_{43}(x, y)$, was set at $w=0.75 \mathrm{~mm}$ and its curvature to $R=2 \mathrm{~m}$. Notice that the input complex field amplitude is generated by applying the same procedure as reported in [20]. For the registration of the fractional power spectra, a CCD camera with resolution $1280 \times 960 \mathrm{px}$ and pixel size $4.6 \mu \mathrm{m}$ has been used.

Antisymmetric FRFT spectra for $N=45$ values of angle from the interval $[\pi / 2,3 \pi / 2]$ have been measured using this system. The Radon-Wigner maps for both separable parts of the field $\mathcal{H}_{4}(x)$ [Fig. 5(a)], and $\mathcal{H}_{3}(y)$ [Fig. 5(b)] have been obtained integrating the measured projections with respect to the $y$ and $x$ coordinates, respectively. Notice that the experimental and the simulated RadonWigner maps [Fig. 5(c) and Fig. 5(d)] are constructed from $N=45$ projections for the same angles.

Applying the inverse Radon transform to the RadonWigner maps, the WDs for $\mathcal{H}_{4}(x)$ and $\mathcal{H}_{3}(y)$ displayed in Fig. 6(a) and Fig. 7(a), are obtained. We observe a good agreement between the experimental and the simulated WD reconstructed from the same number of projections [Fig. 6(b) and 7(b), respectively].

As we have demonstrated by the numerical simulations, the number of projections used for the WD reconstruction plays a crucial role in the phase space tomography method (see Fig. 3 and Fig. 4). Therefore, it is reasonable to suppose that increasing the number of the measured WD projections will lead to even better results. This can be done by a further automatization of the projection acquisition process.

\section{CONCLUSIONS}

We have proposed to use a flexible optical setup, consisting of four cylindrical lenses, to reconstruct the WD of the optical paraxial field separable in Cartesian coordinates. This approach can be applied for both coherent and partially coherent monochromatic light. The numerical simulations and the experimental results demonstrate the feasibility of the method, and their comparison with the theoretical model underscores that the number of measured WD projections plays an important role in the reconstruction process. It clearly indicates that an automatic setup that permits measurement of a large number of WD projections is needed to obtain detailed reconstructions of the WD. For the reconstruction of the WD of nonseparable optical fields, a more sophisticated optical setup is required. These are objectives for our further research.

\section{ACKNOWLEDGMENTS}

The financial support of the Spanish Ministry of Science and Innovation under project TEC 2008-04105/TEC is acknowledged. T. Alieva appreciates the financial support of the Banco Santander Central Hispano and Universidad Complutense de Madrid under project SantanderComplutense, PR-34/07-15914. J. A. Rodrigo acknowledges the Spanish Ministry of Science and Innovation for the "Juan de la Cierva" grant. We also thank G. Cristóbal for his valuable discussions on the numerical calculation of the WD. 


\section{REFERENCES}

1. R. W. Gerchberg and W. O. Saxton, "A practical algorithm for the determination of phase from image and diffraction plane pictures," Optik 35, 237-246 (1972).

2. J. R. Fienup, "Reconstruction of an object from the modulus of its Fourier transform," Opt. Lett. 3, 27-29 (1978).

3. M. R. Teague, "Deterministic phase retrieval: a Green's function solution," J. Opt. Soc. Am. 73, 1434-1441 (1983).

4. K. A. Nugent, "Wave field determination using 3dimensional intensity information," Phys. Rev. Lett. 68, 2261-2264 (1992).

5. M. G. Raymer, M. Beck, and D. F. McAlister, "Complex wavefield reconstruction using phase-space tomography," Phys. Rev. Lett. 72, 1137-1140 (1994).

6. D. F. McAlister, M. Beck, L. Clarke, M. Mayer, and M. G. Raymer, "Optical phase retrieval by phase-space tomography and fractional-order Fourier transformation," Opt. Lett. 20, 1181-1183 (1995).

7. Z. Zalevsky, D. Mendlovic, and R. G. Dorsch, "GerchbergSaxton algorithm applied in the fractional Fourier or the Fresnel domain," Opt. Lett. 21, 842-844 (1996).

8. J. Tu and S. Tamura, "Wave field determination using tomography of the ambiguity function," Phys. Rev. E 42, 1932-1937 (2003).

9. D. Dragoman, "Redundancy of phase-space distribution functions in complex field recovery problems," Appl. Opt. 42, 1932-1937 (2003).

10. T. Alieva, M. Bastiaans, and L. Stankovic, "Signal reconstruction from two close fractional Fourier power spectra," IEEE Trans. Signal Process. 51, 112-123 (2003).

11. X. Liu and K. H. Brenner, "Reconstruction of twodimensional complex amplitudes from intensity measurements," Opt. Commun. 225, 19-30 (2003).

12. A. Semichaevsky and M. Testorf, "Phase-space interpretation of deterministic phase retrieval," J. Opt. Soc. Am. A 21, 2173-2179 (2004).
13. C. Q. Tran, A. G. Peele, A. Roberts, K. A. Nugent, D. Paterson, and I. McNully, "X-ray imaging: a generalized approach using phase-space tomography," J. Opt. Soc. Am. A 22, 1691-1699 (2005).

14. A. V. Gitin, "Optical systems for measuring the Wigner function of a laser beam by the method of phase-spacial tomography," Quantum Electron. 37, 85-91 (2007).

15. U. Gopinathan, G. Situ, T. J. Naughton, and J. T. Sheridan, "Noninterferometric phase retrieval using a fractional Fourier system,” J. Opt. Soc. Am. A 25, 108-115 (2008).

16. H. M. Ozaktas, Z. Zalevsky, and M. A. Kutay, The Fractional Fourier Transform with Applications in Optics and Signal Processing (Wiley, New York, 2001).

17. A. Lohmann, "Image rotation, Wigner rotation, and the fractional Fourier transform," J. Opt. Soc. Am. A 10, 2181-2186 (1993).

18. S. Granieri, W. D. Furlan, G. Saavedra, and P. Andrés, "Radon-Wigner display: a compact optical implementation with a single varifocal lens," Appl. Opt. 36, 8363-8369 (1997).

19. J. A. Rodrigo, T. Alieva, and M. L. Calvo, "Optical system design for orthosymplectic transformations in phase space," J. Opt. Soc. Am. A 23, 2494-2500 (2006).

20. J. A. Rodrigo, T. Alieva, and M. L. Calvo, "Experimental implementation of the gyrator transform," J. Opt. Soc. Am. A 24, 3135-3139 (2007).

21. M. J. Bastiaans, "Application of the Wigner distribution function to partially coherent light," J. Opt. Soc. Am. A 3, 1227-1238 (1986).

22. J. C. Wood and D. T. Barry, "Radon transformation of time-frequency distributions for analysis of multicomponent signals," IEEE Trans. Signal Process. 42, 3166-3177 (1994).

23. S. R. Deans, "Radon and Abel transforms," in The Transforms and Applications Handbook, A. D. Poularikas, ed. (CRC Press, 1999), pp. 8.1-8.95. 